\title{
XXIX. An appendix to the paper on the classes of progressive long waves
}

\section{R. F. Gwyther M.A.}

To cite this article: R. F. Gwyther M.A. (1900) XXIX. An appendix to the paper on the classes of progressive long waves, Philosophical Magazine Series 5, 50:304, 308-312, DOI: $10.1080 / 14786440009463918$

To link to this article: http://dx.doi.org/10.1080/14786440009463918

曲 Published online: 21 Apr 2009.

Submit your article to this journal

Џ Article views: 4

Q View related articles $\square$ 


\section{[ 308 ]}

XXIX. An Appendix to the Paper on the Classes of Progressive Long Waves. By R. F. Gwrther, M.A.*

7 make a rigid test of the method of approximation which 1 I have proposed in my paper on "The Classes of Progressive Long Waves" + , it appears to be desirable to pursue the investigation to a higher order of approximation, especially in the case of the Solitary Wave. For this purpose I continue the expansions in the paper referred to, in which

$$
\left(c^{2}-\frac{g h}{3}\right) h^{2} f^{\prime \prime \prime}=-3 c f^{\prime 2}+2\left(c^{2}-g h\right) f^{\prime}
$$

gave the first approximation.

From these terms we gather the mode in which the order of the terms is to be estimated; namely, that $f^{\prime}$ is of the order $\left(c^{2}-g h\right) / c^{2}$, and $f^{\prime \prime \prime}$ of the order $\left(c^{2}-g h\right)^{2} / c^{4}$. I shall call, in order to : void fractional orders, $f^{\prime}$ of the order 2 of small quantities, $f^{\prime \prime}$ of the order $3 \pm$, and so on,

Acting on this principle, the terms of (3) of the previous paper (the equations in this paper are numbered continuously with those of the former) can readily be arranged in sets of orders 4,6 , and 8 . We thus obtain

$$
\begin{aligned}
& \left(c^{2}-\frac{g h}{3}\right) h^{2} f^{\prime \prime \prime} \\
& -\frac{5 c^{2}-g h}{60} h^{4} f^{\vee}-\frac{g h^{3}}{c} f^{\prime} f^{\prime \prime \prime}+h^{2} f^{\prime \prime 2} \\
& +\left[\frac{2\left(7 c^{2}-g h\right)}{7 !} h^{6} f^{\mathrm{vii}}-\frac{2 c^{2}-g h}{12 c} h^{4} f^{\prime} f^{\vee}-\frac{c h^{4}}{3} f^{\prime \prime} f^{\mathrm{iv}}-\frac{e^{2}+2 g h}{12 c} h^{4} f^{\prime \prime \prime \prime 2}\right. \\
& \left.+h^{2} f^{\prime} f^{\prime \prime 2}-\frac{2 g h^{3}}{c^{2}} f^{\prime 2} f^{\prime \prime \prime}\right] \& \mathrm{c} . \\
& =-3 c f^{\prime 2}+2\left(c^{2}-g h\right) f^{\prime} \\
& +f^{\prime 3} \text {. }
\end{aligned}
$$

The character of the integrals of this equation to the 4th, 6th, and 8th orders are then seen to be as follows:- We shall be able to express in each case $2\left(c^{2}-\frac{g h}{3}\right) h^{2} f^{\prime \prime 2}$ in ascending powers of $f^{\prime}$. The terms of the expansion will proceed as far as the third power in the solution to the 4 th order; as far as the fourth power in the solution to the 6th order; and will extend to the fifth power in the solution to the eighth order;

* Communicated by the Author.

$\dagger$ Phil. Mag. August 1900.

$\ddagger$ This was overlooked in forming the faulty solution (9). 
to the fourth and also to the sixth order the general solution for $f^{\prime}$ will thus be in terms of elliptic functions; but this will cease to be the case to the eighth order. Having drawn these conclusions I shall now omit from (13) the terms (in square brackets) of the eighth order and proceed to the integration to the sixth order.

The solution gives

$$
\begin{aligned}
2\left(c^{2}-\frac{g h}{3}\right) h^{2} f^{\prime \prime 2} & =\mathrm{A}\left(c^{2}-g h\right)^{3}+\mathrm{B}\left(c^{2}-g h\right)^{4} \\
& -\mathrm{A} \frac{15 c^{2}-11 g h}{5\left(c^{2}-\frac{g h}{3}\right)^{2}} c\left(c^{2}-g h\right)^{3} f^{\prime} \\
& +\left(4+\frac{2}{15} \frac{\left(5 c^{2}-g h\right)\left(c^{2}-g h\right)}{\left(c^{2}-\frac{g h}{3}\right)^{2}}\right)\left(c^{2}-g h\right) f^{\prime 2} \\
& -\left(4+\frac{2}{3} \frac{\left(5 c^{2}-g h\right)\left(c^{2}-g h\right)}{\left(c^{2}-\frac{g h}{3}\right)^{2}}\right) c f^{\prime 3} \\
& +4 f^{\prime 4} . . . . . . . . . . .
\end{aligned}
$$

The presence of two constants in this equation is rather apparent than real; for if we take $\mu \frac{c^{2}-g h}{c}$ for the value of $f^{\prime}$ which makes $f^{\prime \prime}$ vanish, we can find both $\mathrm{A}$ and $\mathrm{B}$ in terms of $\mu$. Thus

$$
\mathrm{A}=\frac{4 \mu^{2}(\mu-1)}{c^{2}}
$$

and the equation (14) may be written

$$
\begin{aligned}
& 2\left(c^{2}-\frac{g h}{3}\right) h^{2} f^{\prime{ }_{2}} \\
&=-\frac{4 \mu^{2}(\mu-1)\left(15 c^{2}-11 g h\right)}{5 c\left(c^{2}-\frac{g h}{3}\right)^{2}}\left(c^{2}-g h\right)^{3}\left\{f^{\prime}-\frac{\mu\left(c^{2}-g h\right)}{c}\right\} \\
&+\left(4+\frac{2}{15} \frac{\left(5 c^{2}-g h\right)\left(c^{2}-g h\right)}{\left(c^{2}-\frac{g h}{3}\right)^{2}}\right)\left(c^{2}-g h\right)\left\{f^{\prime 2}-\frac{\mu^{2}\left(c^{2}-g h\right)^{2}}{c^{2}}\right\} \\
&-\left(4+\frac{2}{3} \frac{\left(5 c^{2}-g h\right)\left(c^{2}-g h\right)}{\left(c^{2}-\frac{g h}{3}\right)^{2}}\right) c\left\{f^{\prime 3}-\frac{\mu^{3}\left(c^{2}-g h\right)^{3}}{c^{3}}\right\} \\
&+4\left\{f^{4}-\frac{\mu^{4}\left(c^{2}-g h\right)^{4}}{c^{4}}\right\} . . . . . . .
\end{aligned}
$$


The solution of this equation would give the general motion of a progressive long wave, and would allow us to determine the permissible values of $\mu$. After putting $f^{\prime}=\frac{\mu\left(c^{2}-g h\right)}{c}+\frac{1}{z}$, we obtain an equation which would perhaps be most conveniently treated by Weierstrass's notation, since the factors on the right-hand side are far from obvious. We should be entitled to put $c^{2}=g h$ in the coefficients of the small terms in order to make the expressions simpler. If $c^{2}=g h$ exactly, the constant would have to be treated differently. As, however, my present object is to examine the nature of the approximation which has led to the differential equation, I shall confine the further integration to the case where $\mu=0$, which leads to the case of the solitary wave. In the case of this wave we are able not only to compare our results with the analysis of earlier mathematical investigations, but also with the records of Scott Russell's careful observations.

Putting $\mu=0$ in (15) we obtain

$$
\begin{aligned}
2\left(c^{2}-\frac{g h}{3}\right) h^{2} f^{\prime 2} & =\left(4+\frac{2}{15} \frac{\left(5 c^{2}-g h\right)\left(c^{2}-g h\right)}{\left(c^{2}-\frac{g h}{3}\right)^{2}}\right)\left(c^{2}-g h\right) f^{\prime 2} \\
& -\left(4+\frac{2}{3} \frac{\left(5 c^{2}-g h\right)\left(c^{2}-g h\right)}{\left(c^{2}-\frac{g h}{3}\right)^{2}}\right) c f^{33} \\
& +4 f^{\prime 4} .
\end{aligned}
$$

The first approximation being $f^{\prime}=\frac{c^{2}-g h}{c} \operatorname{sech}^{2} m x$, where $m^{2} h^{2}=\left(c^{2}-g h\right) / 2\left(c^{2}-\frac{g h}{3}\right)$, we are guided to the form of the second approximation, namely,

where

$$
f^{\prime}=p \frac{\operatorname{sech}^{2} m x}{1-q \tanh ^{2} m x}, \text {. . . . . . . . }
$$

$$
\begin{gathered}
m^{2} h^{2}=\frac{c^{2}-g h}{2\left(c^{2}-\frac{g h}{3}\right)}\left(1+\frac{\left(5 c^{2}-g h\right)\left(c^{2}-g h\right)}{30\left(c^{2}-\frac{g h}{3}\right)^{2}}\right), \quad, \quad(1) \\
p=\frac{c^{2}-g h}{c}\left(1+\frac{c^{2}-g h}{c^{2}}-\frac{2\left(5 c^{2}-g h\right)\left(c^{2}-g h\right)}{15\left(c^{2}-\frac{g h}{3}\right)^{2}}\right)
\end{gathered}
$$

and

$$
q=\frac{c^{2}-g h}{c^{2}} .
$$


From this the form of $f$ is easily found ; but the point of most interest is the relation between $m$ and the parameters $c$ and $h$ given by (17).

The method of comparison which I am now about to make was very kindly suggested to me by Sir George Stokes, and is based upon the article communicated by him to Professor Lamb, which appears on p. 421 of the latter's 'Hydro-

dynamics.' simple method to be the necessary relation between $m$ and the parameters in the outskirts of a solitary wave. This result tollows from assuming

$$
\phi=A e^{-2 m(x-c t)} \cos 2 m y
$$

as the form of the velocity-potential when $x$ is large.

Sir George Stokes has now pointed out to me that this is an exact relation provided we admit (1) the possibility of the uniform propagation of the disturbance as a solitary wave, and (2) that in such a wave, at a great distance from the crest, the coefficient of disturbance varies in geometric progression as the distance from the crest increases in arithmetical progression.

If then we admit that $c^{n}=g h \tan 2 m h / 2 m h$ is the exact formula for the wave, being found without assuming any relation between $c$ and $g h$; and if also the conditions of surface pressure can only be everywhere satistied provided

$$
m^{2} h^{2}=\frac{c^{2}-g h}{2\left(c^{2}-\frac{g h}{3}\right)}\left\{1+\frac{\left(5 c^{2}-g h\right)\left(c^{2}-g h\right)}{30\left(c^{2}-\frac{g h}{3}\right)^{2}}\right\},
$$

approximately, the approximate identity of the two formulæ forms the condition for the existence of a Solitary Wave of a certain amplitude. And as under proper restrictions we know that such a wave is capable of propagation, the comparison of the relations under these circumstances is the proper test of the justness of this mode of treatment.

So long as $m h$ is small, we can easily make the comparison by writing the exact formula in the expanded form

$$
\frac{g h}{c^{2}}=1-\frac{4 m^{2} c^{4}}{3 g^{2}}+\frac{1.6 m^{4} c^{8}}{5 g^{4}} \ldots
$$

and substituting in the approximate expression. The expressions are then found to agree identically as far as these three terms in the expansion are concerned. 
Proceeding to consider the larger values of $m h$, to which this expansion for $\frac{g h}{c^{2}}$ becomes inapplicable; Scott Russell's obscrvations led him to the conclusion that the height of the solitary wave might be increased until it became about equal to the depth of the undisturbed fluid; and he gives $c^{2}=2 g h$ as the empiricul formula for the velocity in this case. Making a comparison of the formulæ again with this value for $c^{2}$, the exact formula gives $\tan 2 m h=4 m h$, and

$$
m h=\cdot 583 \text {, approximately. }
$$

The approximate formula in this case gives

$$
m h=\cdot 577 \text {, approximately; }
$$

so that, even in this extreme case, the formulæ are in very close agreement, the difference in the values of $m h$ being just over 1 per cent.

This comparison seems fully to justify the nse of the method I have employed to obtain the approximations.

As the first approximation is that most likely to be employed, it is perbaps worth while to record that the exact and approximate expressions for the relation between $m$ and the parameters are identical for small values of $m h$ if two terms are taken in the expansion for $g h / c^{2}$, and that the difference in the terms of the next order is $2 m^{4} c^{8} / 5 g^{4}$. Taking the extreme case when $c^{2}=2 g h$, the approximate value of $m h$ falls short of the exact value by about 7 per cent.

XXX. The Chemical and Geological History of the Atmosphere. By John Stevenson, M.A., F.I.C.*

$$
\text { I.-The History of Free Oxygen. }
$$

TWEE question as to whether any notable change has taken place in the chemical composition of the atmosphere in the course of geological history is one that has received a considerable amount of attention from chemists and geologists, though probably not so much as the importance and interest of the subject deserve. It has been sufficiently clear to geologists for many years that the carbon of our coal supplies has been derived from carbonic acid formerly diffused through the atmosphere or dissolved in the ocean ; and that if all this carbonic acid existed at one time in the atmosphere, the atmosphere must have been very much richer in carbonic * Communicated by Prof. G. F. FitzGerald, F.R.S. 